\title{
Chemodynamical models of barred galaxies
}

\author{
Hervé Wozniak ${ }^{1}$ and Léo Michel-Dansac ${ }^{2} \dagger$ \\ ${ }^{1}$ Université de Lyon 1, Centre de Recherche Astronomique de Lyon, Lyon, France \\ email: herve.wozniak@obs.univ-lyon1.fr \\ ${ }^{2}$ UNAM, Instituto de Astronomia, Ensenada, Mexico \\ email: leo@astrosen.unam.mx
}

\begin{abstract}
Chemodynamical disc galaxy simulations calibrated with stellar populations synthesis models allow to build mock multi-wavelength images from numerical simulations. The morphological evolution of the mass and colour distributions can thus be simultaneously followed. We present the evolution of the stellar disc and bar structural parameters and summarize a number of important results we got in the recent past.
\end{abstract}

Keywords. galaxies: kinematics and dynamics, galaxies: spiral

Since the first pioneering N-body simulations till the most recent ones, morphological evolution of disc galaxy simulations have focused on the properties of the mass distribution. To make detailed comparisons between these models and observations it is implicitly assumed that all particles have the same mass-to-light ratio taken as unity for convenience. However, it is well known that real galaxies are built of composite stellar populations, thus of various mass-to-light ratios and metallicities, leading to a complex distribution of apparent stellar ages (Wozniak 2007). Moreover, dust extinction is not uniform and then does not affect in the same way the various stellar populations.

Thus, to make more straightforward comparisons of simulations with multi-wavelength observations, we have used stellar population synthesis models (Bruzual \& Charlot 1993) to photometrically calibrate our N-body/hydro simulations which include stars, gas and star formation. Details on the code, numerical simulations, spectrophotometric calibration technique, mock images computing, and dust extinction modelling can be found in Michel-Dansac \& Wozniak (2004). We hereafter summarise the results presented in recent papers (Michel-Dansac \& Wozniak, 2004, Michel-Dansac \& Wozniak, 2006, MichelDansac \& Wozniak, 2007, Wozniak \& Michel-Dansac, 2007).

The typical evolution of our simulations is as follows: the initial disc quickly develops a strong bar and a spiral structure both in the stellar and the gaseous components. Star formation (SF) is not homogeneously distributed over the whole disc but is concentrated along the bar major axis and along the spiral arms. The main peak of SF is associated to the phase of bar formation, followed by a number of secondary peaks. The global SFR reaches $30 \mathrm{M}_{\odot} \mathrm{yr}^{-1}$. Typical SFR density ranges from $7.310^{-4}$ (in the disc) to $365 \mathrm{M}_{\odot} \mathrm{yr}^{-1} \mathrm{kpc}^{-2}$ (in the nucleus). These values are in good agreement with Kennicutt (1998). The next peaks are the result of the gas inflow towards the central region of the disc. Indeed, the gravitational torques applied by the bar and the spiral arms on the gas create several regions of very high gas density in which SF is triggered. Such inflow is not continuous, but rather proceeds in bursts.

A dynamically self-consistent SFR peak produces a large increase in total luminosity in all wavebands whereas the stellar mass of the new population is much lower than the

$\dagger$ Present address: Observatorio Astronómico de Córdoba and CONICET, Laprida 854, X5000BGR, Córdoba, Argentina. 
underlying old stellar mass. However, luminosity peaks can be almost completely obscured by dust extinction, since the young stellar population initially seats in gas-rich regions where extinction is maximum. This effect is particularly important on $\mathrm{B}-\mathrm{H}$ colour maps since regions of highest SFR could become unobservable. It could lead to underestimate SFR from photometric or colour measurements. For instance, the luminosity integrated into the bar region reaches $\approx 80 \%$ of the total B band luminosity at the SFR maximum in absence of dust, and $>60 \%$ when SFR is low. When SFR is maximum less than $20 \%$ of the B band luminosity of the bar region succeed to escape in the presence of dust because most star formation occurs in gas-rich regions where extinction is the most efficient.

Isophotal radii evolve with respect to mass redistribution during the dynamical evolution but also with respect to stellar evolution. Hence, the dynamical properties of the area enclosed by any isophotal radius depends on the waveband and on the SF activity. Extinction effects do not affect the determination of isophotal radii, even in $\mathrm{B}$ band. It is also noteworthy that the luminosity integrated inside the isophotal radius at 20.5 mag $\operatorname{arcsec}^{-2}$ in the $\mathrm{H}$ band is not a good tracer of mass.

An accurate determination of the bar length is crucial in several observational or theoretical analysis. We indeed gave evidence that the ratio $\mathcal{R}=R_{\text {Corotation }} / R_{\text {bar }}$ could increase from 1 (fast bar) to 1.4 (slow bar) just by changing the criterion used to estimate bar lengths. Most methods to estimate the bar length rely on the shape of isophotes and their radial profiles. However, as long as SF is active, mass isodensities in the bar region are rounder than isophotes leading to very different ellipticity profiles for surface brightness and mass distribution. Thus, the surface brightness distribution, even in $\mathrm{H}$ band, is a good tracer of mass only when SFR is below $1 \mathrm{M}_{\odot} \mathrm{yr}^{-1}$. The radius of the maximum ellipticity $\left(e^{\max }\right)$, often used to determine bar length, depends strongly on the waveband. Within a typical error of $10 \%$, bar lengths determined with other criteria than the one related to $e^{\max }$ are not colour dependant. The situation is slightly different when dust absorption is taken into account. Criteria giving the smallest bar lengths are much more affected than others. This is again the case of the $e^{\max }$ position. Another noteworthy effect is that $e^{\max }$ decreases with time, as does the bar strength $Q_{b}$, but not at the same rate than $Q_{b}$. The use of $e^{\max }$ to estimate the bar strength could thus lead to severe errors, especially in a comparison from one galaxy to another.

In general, for projection angles $i$ between 15 and $70^{\circ}$, the bar length is underestimated with respect to its face-on value. However for $i<30^{\circ}$ errors remain below $20 \%$. Measurements also depends on the resolution but errors never exceed $15 \%$ when resolution is downgraded by a factor 5 .

Finally, we also showed that the minimum of ellipticity located just outside the bulk of the bar is correlated with the Corotation radius whereas the location of Ultra Harmonic Resonance can be approximated using the end of the position-angle plateau of bar isophotes. These criteria give thus a good approximation of resonance positions.

\section{References}

Bruzual, G., \& Charlot, S., 1993, ApJ 405, 538

Michel-Dansac\& L., \& Wozniak, H., 2004, A\&A 421, 863

Michel-Dansac, L., \& Wozniak, H., 2006, A\&A 452, 97

Michel-Dansac, L. \& Wozniak, H., 2007, in Chemodynamics: from the first stars to local galaxies, CRAL Conference Series I, held in Lyon 10-16 July 2007.

Kennicutt, R.C., 1998, ARA\&A 36, 189

Wozniak, H., \& Michel-Dansac, L. 2007, in Chemodynamics: from the first stars to local galaxies, CRAL Conference Series I, held in Lyon 10-16 July 2007.

Wozniak, H., 2007 A\&A accepted 